\title{
Effects of weekly pain monitoring on back pain outcomes: a non-randomised controlled study
}

Alice Kongsted ${ }^{1,2^{*}}$ (1) Tue Secher Jensen ${ }^{1,2,3}$, Klaus Doktor $^{1,2}$ and Lise Hestbæk ${ }^{1,2,3}$

\begin{abstract}
Background: Disease monitoring is an important element of self-management of several chronic diseases. Pain monitoring has become very easily available, but the role in musculoskeletal pain conditions is not clear. Awareness of pain might be helpful for people to understand pain, but focusing on pain may on the contrary negatively affect pain experience and behaviours. The objective of this study was to investigate the potential impact of pain monitoring on low back pain (LBP), specifically to determine if pain intensity, activity limitation and pain control, differed between patients with weekly pain monitoring over 12 months and patients with follow-ups at 2 weeks, 3 months and 12 months.

Methods: This was a non-randomised controlled study embedded in a cohort study with data collection November 1st 2016 to December 21st 2018. Adults seeking care for LBP were enrolled at the first visit to a chiropractor and followed with surveys after 2 weeks, 3 months and 12 months. Those enrolled first, $n=1,623$, furthermore received weekly SMS-questions about pain frequency and pain intensity, whereas those enrolled next was the control group, $n=1,269$ followed only by surveys. Outcomes at 12 -months were compared, adjusting for group differences on baseline parameters.
\end{abstract}

Results: LBP intensity (0-10) was slightly lower at 12-months follow-up in the SMS group than the control group (adjusted beta $-0.40(95 \% \mathrm{Cl}:-0.62 ;-0.19))$. No relevant between-group differences were observed for activity limitation (0-100) $(1.51(95 \% \mathrm{Cl}:-0.83 ; 3.85))$ or ability to control pain $(0-10)(-0.08(95 \% \mathrm{Cl}-0.31 ; 0.15))$.

Conclusions: Frequent pain monitoring did not demonstrate any negative effects of weekly pain monitoring, and it was perhaps even helpful. The role of self-monitoring as part of self-managing LBP should be explored further including optimal frequencies, formats, and methods for feedback.

Trial registration: The study was not registered as a clinical trial.

Keywords: Back pain, Data collection, Monitoring, Pain measurement, Self-management

\section{Background}

Non-communicable diseases constitute a large part of the global burden of disease with musculoskeletal conditions being among the main causes for disability [1,

\footnotetext{
*Correspondence: akongsted@health.sdu.dk

${ }^{1}$ Chiropractic Knowledge Hub, Campusvej 55, 5230 Odense M, Denmark

Full list of author information is available at the end of the article
}

2]. People living with persistent or recurrent conditions need knowledge, skills, and tools to manage their health conditions in everyday life [3]. For many people this involves contact with health care providers, but most of the time people manage health conditions on their own or with assistance from family and friends.

Self-management may involve engagement in health promoting activities, symptom management and original author(s) and the source, provide a link to the Creative Commons licence, and indicate if changes were made. The images or other third party material in this article are included in the article's Creative Commons licence, unless indicated otherwise in a credit line to the material. If material is not included in the article's Creative Commons licence and your intended use is not permitted by statutory regulation or exceeds the permitted use, you will need to obtain permission directly from the copyright holder. To view a copy of this licence, visit http://creativecommons.org/licenses/by/4.0/. The Creative Commons Public Domain Dedication waiver (http://creativeco mmons.org/publicdomain/zero/1.0/) applies to the data made available in this article, unless otherwise stated in a credit line to the data. 
self-monitoring of the condition [4]. Self-monitoring of physiological measures such as blood glucose levels in diabetes and blood pressure in hypertension are important for individual health decisions. In musculoskeletal pain conditions, the role of disease monitoring is less clear, as there are no objective measures to inform actions. Musculoskeletal pain often varies considerably from day to day, and monitoring may be helpful for people to make sense of their pain [5]. Also, frequent pain measures are used in research to evaluate the course of pain as trajectories of pain are not well captured by the few follow-ups commonly used $[6,7]$.

Self-monitoring of pain has become easily available and was an integrated feature in 11 out of 19 and 14 out of 28 pain management apps identified in two recent reviews $[8,9]$. This awareness of pain might be helpful for people to understand the variation over time and reflect on how the pain relates to other aspects of life [5]. However, a continuous focus on pain might also negatively affect pain intensity and pain behaviours, as expecting pain and focusing on pain entail a potential for affecting pain perception negatively [10]. It is thus important to consider if self-monitoring as part of interventions could be harmful. In research it is also important to know if the frequent measuring of pain affects outcomes differently than using few follow-ups over the same time period.

Currently there is very sparse evidence of the potential effects of pain monitoring on patient outcomes. A systematic review of effectiveness of digital support interventions for self-management of back pain did not identify studies providing evidence for or against selfmonitoring of pain [11]. In a previous explorative study, we reported outcomes in patients with back pain and in school children who participated in weekly SMS-monitoring of pain as compared to similar samples with single time point follow-ups, and observed slightly better outcome with weekly pain measurements than in those without. However, there was a considerable risk that samples were not comparable on unmeasured factors [12].

The objective of this study was to determine if outcomes, in terms of pain intensity, LBP related activity limitation and pain control, differed between patients with weekly pain monitoring over 12 months and patients with follow-ups at 2 weeks, 3 months and 12 months only.

\section{Method}

This was a non-randomised controlled study embedded in the Danish Chiropractic Low Back Pain Cohort (ChiCo). All patients included were invited to complete baseline and follow-up questionnaires (see "Data Collection" below). Weekly SMS-tracking of pain in addition to follow-up questionnaires was planned for the first patients enrolled in the cohort until a level of 1,000 participants actively answering the text messages was reached, while the subsequent participants were followed by questionnaires only. Treatment was not affected by study participation, and the treating chiropractors were blinded to the patient reported information registered in the study. The ChiCo cohort and procedures for recruitment and data collection have previously been described in detail [13]. The protocol for this study was not pre-registered. All methods were carried out in accordance with relevant guidelines and regulations.

\section{Setting}

Participants were recruited from a convenience sample of ten Danish chiropractic clinics. Most patients selfrefer to chiropractic treatment in Denmark. Around 20\% of costs of chiropractic care is covered by the national health insurance, while the rest is paid out of pocket, by the patients' private insurance, or some combination of both.

\section{Participants}

Adults ( $>18$ years of age) consulting with a primary complaint of LBP with or without leg pain were eligible for inclusion. People who either could not read Danish, were in ongoing treatment for LBP, or with suspected serious pathology were not invited to the study.

\section{Allocation to SMS-tracking}

Based on a sample size estimation for investigating trajectories of LBP (unrelated to this study), 1,000 participants with weekly data collection using SMS-tracking was needed. Because of considerable costs related to the set-up of SMS-tracking, this was planned for the participants first enrolled, and to compensate for project participants without a mobile phone and expected dropouts, 1,623 were enrolled in the SMS-tracking sample (hereafter referred to as the SMS group). Those recruited subsequently were not followed with SMS-tracking and constituted the control group (Fig. 1). We included the entire sample in the study which would be sufficient to demonstrate even a very small effect size.

\section{Content of SMS-tracking}

The SMS group received text messages to their mobile phone every week starting seven days after enrolment. The questions asked were (1) "How many days have you had back pain (or back-related leg pain) within the last 7 days? (please answer with one number from 0 to 7 )"; (2) "How severe was the pain typically on a scale from 0 to 10 ?"; and (3) "How many days were you home last week from work or study because of your back pain? (please answer with one number from 0 to 7 )". In case the answer 


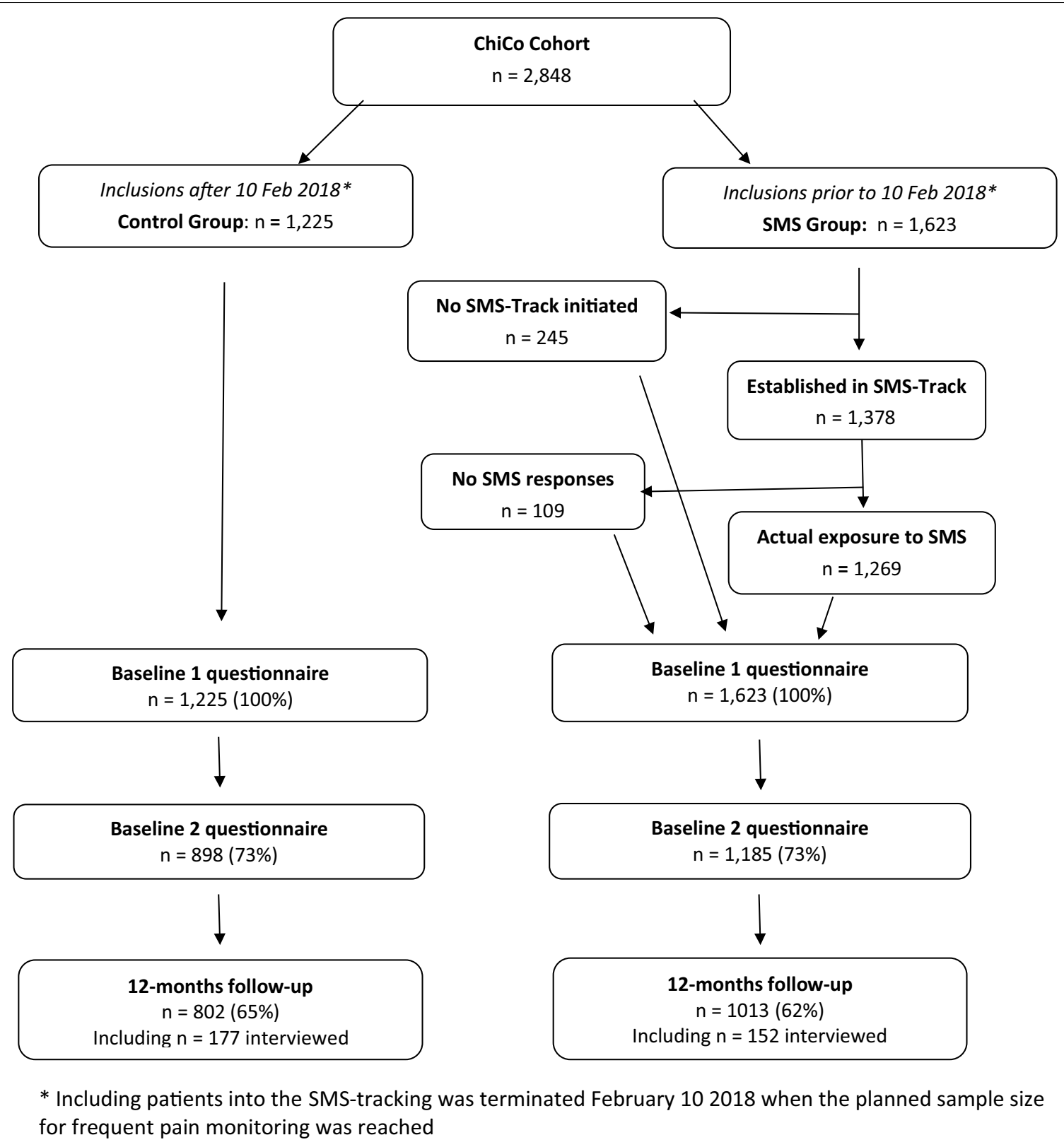

Fig. 1 Study flowchart

to the first message was "0" the two following messages were not sent. If no response was registered within 2 days, participants received one automated reminder.

\section{Data collection in both groups}

Questionnaire data were collected and stored using the online system Research Electronic Data Capture (REDCap) hosted by the Odense Patient data Explorative Network (OPEN). Record IDs and mobile phone numbers were exported to an SMS-Track service that automated weekly distribution of text message questions. Baseline data was collected in two parts. The initial part via an iPad in the clinic prior to the consultation and the second part via a link to an online survey sent by e-mail to the participant on the day of enrolment. Links to follow-up questionnaires were sent after 2 weeks, 3 months, and 12 months. Timing and content of the questionnaires did not differ between the SMS group and the control group. 


\section{Outcome measures}

The primary outcome measure for this study was LBP intensity at 1-year follow-up. Activity limitation and pain control after 1 year were secondary outcomes.

LBP intensity was measured on a $0-10$ numeric rating pain scale $(0=$ no pain; $10=$ worst imaginable pain $)$ asking about typical pain in the previous week [14, 15]. Moderate to severe pain at follow-up was defined as NRS $>3$ [16].

Activity limitation was measured by the 23-item Roland Morris Disability Questionnaire (RMDQ) converted to a proportional sum-score ranging from 0 to $100(0=$ No disability; $100=$ Fully disabled $)[15$, 17]. Missing items in partly completed RMDQs were imputed by multiple imputation prior to calculating the sum-score. Non-improvement on RMDQ was defined as $<30 \%$ reduction in the sum score from baseline to 1-year follow-up [18].

Pain control was measured by a single coping item from the Örebro Musculoskeletal Pain Questionnaire (ÖMPQ) (Danish version) asking "Given an average day, to what extent can you handle or control your pain?" $(0=$ Not at all; $10=$ Complete control) [19]. As no formal cut-point exists for categorisation of the item, we defined "lack of control" as scoring $<5$. The ÖMPQ was developed as a prognostic screening tool and this measure of pain control has not been validated as an outcome measure.

\section{Baseline variables}

The two study groups were compared at baseline on the following parameters in addition to baseline values of outcome measures: Age (years), sex, education (higher or further education, vocational education, no qualifying education, other education), physical load at work (very strenuous, strenuous, somewhat strenuous, light, very light), episode duration, STarT Back Tool risk profile (low, medium, high risk), and recovery expectation (ÖMPQ "How certain are you that you will be able to return to ALL of your usual activities 1 month from today?").

\section{Analyses}

Patient characteristics were described as means with standard deviations (SD) and proportions (\%).

Potential differences between the SMS group and the control group in the characteristics of those not completing 12-months follow-up were investigated in logistic regression models with SMS group, baseline characteristic (age, sex, LBP intensity, activity limitation, pain control, STarT risk profile, episode duration, recovery expectations) and an interaction between the two as independent variables and drop out as the dependent variable.

Group differences were investigated using the "intention to treat" principle, with all patients invited to SMS-tracking analysed in the SMS group even if not responding to any SMS questions. In a sensitivity analysis we included only those responding to SMS in more than 26 weeks, i.e. more than half of the SMS follow-ups, in the SMS group. The control group did not differ between the two analysis.

Group differences were estimated by linear (continuous outcomes) and logistic (binary outcomes) mixed models with clinician as random effects to account for dependence between observation from the same chiropractor and reported as beta coefficients and Odds Ratios (OR) with 95\% confidence intervals (CI). Group differences were reported as crude estimates and after adjustment for baseline values that were observed to differ slightly between groups: LBP intensity, age, sex, duration of present episode, educational level, and workload.

Analyses were performed using Stata 16.1 (StataCorp, Texas, USA).

\section{Results}

\section{Study participants and response rates}

ChiCo cohort recruitment was initiated on November 1st, 2016. Participants enrolled prior to February 10th, 2018 totaling 1,623, formed the SMS group, while the remaining 1,225 participants enrolled until the cohort closure on December 21st, 2018 formed the control group.

Demographic parameters including age, sex, BMI and employment did not differ substantially between the groups (Table 1). Compared to the control group, a slightly larger proportion of participants in the SMS group reported a higher education ( $54 \%$ vs. $49 \%$ ) a light or very light workload (62\% vs. $58 \%$ ), and more participants in the SMS group, and a very short duration of the present episode of pain ( 49 vs. $44 \%$ ). The two groups had very similar baseline values of LBP intensity, activity limitation, pain control and recovery expectations (Table 1).

In both groups, a response rate of $73 \%$ for the second part of the baseline questionnaire was obtained, while the SMS group had slightly lower response rates at 12-months follow (Fig. 1). Most baseline characteristics were not associated with non-response to 12-months follow-up, and the associations did not differ substantially between the groups (Table 2).

\section{Effect of SMS-tracking}

The primary outcome LBP intensity was slightly lower in the SMS group than the control group (mean 2.1 vs. 2.6 ; adjusted beta -0.40 (95\% CI: $-0.62 ;-0.19))$. This 
Table 1 Patient characteristics at baseline

\begin{tabular}{|c|c|c|}
\hline Patient characteristics at baseline & $\begin{array}{l}\text { SMS group } \\
(N=1,623)\end{array}$ & $\begin{array}{l}\text { Control group } \\
(\mathrm{N}=1,225)\end{array}$ \\
\hline Age; mean (SD, range) & $44.5(13.6 ; 18-80)$ & $44.7(13.8 ; 18-87)$ \\
\hline Sex, female; n (\%) & $655(40.4 \%)$ & $512(41.8 \%)$ \\
\hline BMl; mean (SD) & $26.7(4.9)$ & $27.0(5.1)$ \\
\hline Employment, yes; n (\%) & $1302(82.4 \%)$ & $985(81.8 \%)$ \\
\hline \multicolumn{3}{|l|}{ Longest education } \\
\hline Higher or further education; n (\%) & $615(53.5 \%)$ & $425(48.8 \%)$ \\
\hline Vocational education; n (\%) & $318(27.7 \%)$ & $276(31.7 \%)$ \\
\hline No qualifying education; $\mathrm{n}(\%)$ & $174(15.1 \%)$ & $139(16.0 \%)$ \\
\hline Other education; n (\%) & $43(3.7 \%)$ & $31(3.6 \%)$ \\
\hline \multicolumn{3}{|l|}{ Workload } \\
\hline Very strenuous; n (\%) & $19(2.1 \%)$ & $18(2.6 \%)$ \\
\hline Strenuous; n (\%) & $108(11.9 \%)$ & $84(12.1 \%)$ \\
\hline Somewhat strenuous; n (\%) & $216(23.8 \%)$ & $186(26.9 \%)$ \\
\hline Light; $\mathrm{n}(\%)$ & $281(30.9 \%)$ & $225(32.5 \%)$ \\
\hline Very light; $n$ (\%) & $285(31.4 \%)$ & $179(25.9 \%)$ \\
\hline Previous episodes with low back pain, yes; n (\%) & $934(82.4 \%)$ & $718(84.4 \%)$ \\
\hline Previous treatment for low back pain, yes; $\mathrm{n}(\%)$ & $763(65.7 \%)$ & $594(67.4 \%)$ \\
\hline Back pain intensity, NRS (0-10); mean (SD) & $6.7(2.1)$ & $6.7(2.1)$ \\
\hline \multicolumn{3}{|l|}{ Episode duration } \\
\hline 1-7 days; $n(\%)$ & $793(49.4 \%)$ & $535(43.8 \%)$ \\
\hline $1-4$ weeks; n (\%) & $359(22.3 \%)$ & $315(25.8 \%)$ \\
\hline 1-3 months; $\mathrm{n}(\%)$ & $169(10.5 \%)$ & $163(13.4 \%)$ \\
\hline >3 months; $\mathrm{n}(\%)$ & $286(17.8 \%)$ & $208(17.0 \%)$ \\
\hline \multicolumn{3}{|l|}{ Prognostic index, STarT ${ }^{a}$} \\
\hline Low; n (\%) & $657(42.3 \%)$ & $513(43.4 \%)$ \\
\hline Medium; n (\%) & $558(35.9 \%)$ & $409(34.6 \%)$ \\
\hline High; n (\%) & $340(21.9 \%)$ & $260(22.0 \%)$ \\
\hline Activity limitation, RMDQ (0-100); mean (SD) ${ }^{b}$ & $55.6(23.8)$ & $54.1(24.0)$ \\
\hline Ability to control pain, NRS (0-10); mean (SD) & $5.5(2.4)$ & $5.5(2.3)$ \\
\hline Recovery expectations, NRS (0-10); mean (SD) & $7.4(3.0)$ & $7.5(2.9)$ \\
\hline
\end{tabular}

NRS, Numeric Rating Scale; RMDQ, Roland Morris Disability Questionnaire; STarT,The Keele STarT Back Screening Tool; SD, Standard deviation

${ }^{\text {a }}$ Missings imputed if at least 7 out of 9 STarT items were answered.

${ }^{\mathrm{b}}$ Missings imputed for partly completed RMDQ

was also reflected in a lower proportion with pain intensity $>3$ in the SMS group than in the control group $(23.3 \%$ vs. 29.9\%; adjusted OR 0.70 (95\% CI: 0.56; 0.88)) (Tables 3 and 4). Secondary outcomes did not differ importantly between groups but showed some imprecision (Tables 3 and 4).

\section{Sensitivity analysis}

Of the 1,623 participants in the SMS group, 245 did not provide mobile phone information and another 109 did not answer any of the text messages. The group of participants actually responding to SMS tracking thus consisted of 1,269 participants of whom 995 (78\%) answered more than 26 of the 52 text messages (median
51 responses (IQR 38-52)). Within the SMS group, we compared baseline characteristics of participants answering more than half the text messages, less than half, or none at all. Females, older participants, and patients with a short duration of the present episode, or a STarT low risk profile more often responded to more than half of the SMS questions, whereas baseline values of LBP intensity, activity limitation and ability to control pain were almost the same across the three responder groups (Table 5 ).

Only including those who responded to more than 26 out of 52 SMS messages in the group comparisons revealed results similar to the intention to treat analysis (Tables 3 and 4). 
Table 2 Drop-out analysis

\begin{tabular}{|c|c|c|c|}
\hline Patient characteristics at baseline & $\begin{array}{l}\text { SMS group } \\
\text { OR }(95 \% \mathrm{Cl}) \\
N=1,013 \text { responders } \\
N=610 \text { non-responders }\end{array}$ & $\begin{array}{l}\text { Control group } \\
\text { OR }(95 \% \mathrm{Cl}) \\
N=802 \text { responders } \\
N=423 \text { non-responders }\end{array}$ & $\begin{array}{l}\text { Interaction } \\
\text { between group and } \\
\text { characteristic } \\
\text { OR (p-value) }\end{array}$ \\
\hline Age (years) & $0.98(0.97-0.99)$ & $0.97(0.96-0.98)$ & $1.01(p=0.1)$ \\
\hline Female (ref. male) & $0.99(0.80-1.21)$ & $0.80(0.63-1.02)$ & $1.23(p=0.2)$ \\
\hline Back pain intensity, NRS (0-10) & $1.01(0.96-1.06)$ & $1.02(0.96-1.08)$ & $0.99(p=0.8)$ \\
\hline Activity limitation, RMDQ (0-100) & $1.00(1.00-1.00)$ & $1.00(1.00-1.01)$ & $1.00(p=0.8)$ \\
\hline Ability to control pain, NRS (0-10) & $0.92(0.88-0.96)$ & $0.94(0.89-0.99)$ & $0.98(p=0.5)$ \\
\hline Recovery expectations, NRS (0-10) & $0.98(0.94-1.01)$ & $0.98(0.94-1.02)$ & $0.99(p=0.8)$ \\
\hline \multicolumn{4}{|l|}{ STarT risk profile } \\
\hline \multicolumn{4}{|l|}{ Low (ref) } \\
\hline Medium & $1.09(0.86-1.39)$ & $0.95(0.72-1.25)$ & $1.15(p=0.4)$ \\
\hline High & $1.49(1.14-1.95)$ & $1.36(1.00-1.85)$ & $1.10(p=0.6)$ \\
\hline \multicolumn{4}{|l|}{ Episode duration } \\
\hline \multicolumn{4}{|l|}{$<1$ week (ref) } \\
\hline $1-4$ weeks & $0.92(0.71-1.19)$ & $0.96(0.71-1.29)$ & $0.95(p=0.8)$ \\
\hline $1-3$ months & $0.79(0.55-1.12)$ & $0.73(0.50-1.08)$ & $1.08(p=0.8)$ \\
\hline$>3$ months & $1.13(0.86-1.49)$ & $1.41(1.02-1.96)$ & $0.80(p=0.3)$ \\
\hline
\end{tabular}

Odds ratios for non-response at 12 months follow-up for the SMS group and the control group

$\mathrm{NRS}=$ Numeric Rating Scale; RMDQ = Roland Morris Disability Questionnaire; STarT = The Keele STarT Back Screening Tool; OR=Odds ratio; $\mathrm{Cl}=$ confidence interval; ref $=$ reference group

Table 3 Observed outcomes in the control group, the total SMS group, and the participants in the SMS group responding in more than 26 out of the 52 weeks

\begin{tabular}{|c|c|c|c|c|}
\hline \multicolumn{2}{|l|}{ Continuous outcomes } & Control group $(\mathrm{N}=1,225)$ & SMS group $(N=1,623)$ & $\begin{array}{l}\text { SMS group responding } \\
\text { in }>26 \text { weeks }(N=995)\end{array}$ \\
\hline \multicolumn{2}{|c|}{ LBP at 12 months follow-up, NRS (0-10); mean (SD) } & $2.6(2.4)(n=799)$ & $2.1(2.4)(n=1,012)$ & $2.0(2.4)(n=826)$ \\
\hline \multicolumn{2}{|l|}{ Activity limitation, RMDQ (0-100); mean (SD) } & $19.5(22.8)(n=610)$ & $20.1(22.9)(n=847)$ & $19.9(22.6)(n=720)$ \\
\hline \multicolumn{2}{|l|}{ Ability to control pain, NRS (0-10); mean (SD) } & $7.9(2.4)(n=790)$ & $7.9(2.6)(n=999)$ & $7.9(2.6)(n=813)$ \\
\hline Binary outcomes & \multicolumn{2}{|c|}{ Control group $(\mathrm{N}=1,225)$} & up $(N=1,623)$ & $\begin{array}{l}\text { SMS group responding } \\
\text { in }>26 \text { weeks }(N=995)\end{array}$ \\
\hline Pain > 3, yes; n (\%) & \multicolumn{2}{|c|}{$239(29.9 \%)(n=799)$} & $3 \%)(n=1,012)$ & $182(22.0 \%)(n=826)$ \\
\hline $\begin{array}{l}\text { Non-improvement of activity limitation; } \\
\mathrm{n}(\%)\end{array}$ & \multicolumn{2}{|c|}{$137(22.8 \%)(n=601)$} & \%) $(n=835)$ & $152(21.3 \%)(n=714)$ \\
\hline Lack of pain control, yes; $\mathrm{n}(\%)$ & \multicolumn{2}{|c|}{$85(10.8 \%)(n=790)$} & 5\%) $(n=999)$ & $90(11.1 \%)(n=81)$ \\
\hline
\end{tabular}

$\mathrm{LBP}=$ low back pain; NRS = Numeric Rating Scale; RMDQ = Roland Morris Disability Questionnaire; $\mathrm{SD}=$ standard deviation

\section{Discussion}

\section{Principal findings}

In this large non-randomised controlled study nested within the Danish ChiCo, we found a slightly lower pain intensity at 12 months follow-up in LBP patients who had provided weekly reports of their LBP through SMS-tracking, compared to patients who had not reported pain through SMS-tracking. The magnitude of the observed positive effect does not seem clinically relevant as a treatment effect, but provides evidence of weekly pain monitoring not having harmful effects on pain outcomes. There were no between-group differences observed in relation to activity limitation or pain control.

These findings align well with the results from a previous study also demonstrating lower odds for pain intensity above 3/10 at follow-up in the SMS-group than in the control group, but no differences in frequency, activity limitation and bothersomeness [12]. However, data used in that study were not optimally suited for the purpose of studying effects of frequent monitoring and the sample was small and suffered substantially from drop-out. 
Table 4 Group differences at 12-months follow-up

\begin{tabular}{|c|c|c|c|c|}
\hline \multirow[t]{2}{*}{ Continuous outcomes } & \multicolumn{2}{|c|}{$\begin{array}{l}\text { SMS group versus control group } \\
\text { Main analysis }\end{array}$} & \multicolumn{2}{|c|}{$\begin{array}{l}\text { SMS group responding }>26 \text { weeks versus } \\
\text { control group } \\
\text { Sensitivity analysis }\end{array}$} \\
\hline & $\begin{array}{l}\text { Crude } \\
\beta(95 \% \mathrm{Cl})\end{array}$ & $\begin{array}{l}\text { Adjusted } \\
\beta(95 \% \mathrm{Cl})\end{array}$ & $\begin{array}{l}\text { Crude } \\
\beta(95 \% \mathrm{Cl})\end{array}$ & $\begin{array}{l}\text { Adjusted } \\
\beta(95 \% \mathrm{Cl})\end{array}$ \\
\hline $\begin{array}{l}\text { LBP at } 12 \text { months follow-up, NRS } \\
(0-10) ; \text { mean (SD) }\end{array}$ & $-0.42(-0.65 ;-0.19)$ & $-0.40(-0.62 ;-0.19)$ & $-0.48(-0.73 ;-0.24)$ & $-0.44(-0.68 ;-0.21)$ \\
\hline Activity limitation, RMDQ (0-100) & $1.12(-1.34 ; 3.58)$ & $1.51(-0.83 ; 3.85)$ & $0.88(-1.68 ; 3.45)$ & $1.30(-1.12 ; 3.73)$ \\
\hline Ability to control pain, NRS (0-10) & $-0.05(-0.28 ; 0.19)$ & $-0.08(-0.31 ; 0.15)$ & $-0.10(-0.36 ; 0.15)$ & $-0.12(-0.37 ; 0.13)$ \\
\hline Binary outcomes & $\begin{array}{l}\text { Crude } \\
\text { OR }(95 \% \mathrm{Cl})\end{array}$ & $\begin{array}{l}\text { Adjusted } \\
\text { OR }(95 \% \mathrm{Cl})\end{array}$ & $\begin{array}{l}\text { Crude } \\
\text { OR }(95 \% \mathrm{Cl})\end{array}$ & $\begin{array}{l}\text { Adjusted } \\
\text { OR }(95 \% \mathrm{Cl})\end{array}$ \\
\hline Pain $>3$ & $0.71(0.58 ; 0.88)$ & $0.70(0.56 ; 0.88)$ & $0.66(0.53 ; 0.83)$ & $0.65(0.50 ; 0.83)$ \\
\hline $\begin{array}{l}\text { Non-improvement of activity limita- } \\
\text { tion }\end{array}$ & $1.00(0.77 ; 1.31)$ & $1.02(0.77 ; 1.34)$ & $0.94(0.71 ; 1.24)$ & $0.99(0.72 ; 1.28)$ \\
\hline Lack of pain control & $0.97(0.72 ; 1.32)$ & $1.00(0.74 ; 1.36)$ & $1.03(0.76 ; 1.41)$ & $1.05(0.76 ; 1.45)$ \\
\hline
\end{tabular}

Adjusted for back pain at baseline, age, sex, episode duration, education and workload. LBP = low back pain; NRS = Numeric Rating Scale; RMDQ = Roland Morris Disability Questionnaire; SD = standard deviation

Table 5 Patient characteristics of the SMS group divided into subgroups responding to SMS questions in $>26$ weeks (more than half of SMS messages); 1-26 weeks, 0 weeks

\begin{tabular}{|c|c|c|c|}
\hline \multirow[t]{2}{*}{ Patient characteristics at baseline } & \multicolumn{3}{|l|}{ SMS group } \\
\hline & $>26$ responses $(n=995)$ & $1-26$ responses $(n=274)$ & 0 responses $(n=354)$ \\
\hline Age; mean (SD) & $45.6(13.0)$ & $41.9(14.8)$ & $43.5(14.2)$ \\
\hline Sex, female; n (\%) & $420(42.2 \%)$ & $103(37.6 \%)$ & $132(37.3 \%)$ \\
\hline Back pain intensity, NRS (0-10); mean (SD) & $6.7(2.0)$ & $6.7(2.1)$ & $6.6(2.1)$ \\
\hline Episode duration, > 7 day; $\mathrm{n}$ (\%) & $478(48.6 \%)$ & $148(54.4 \%)$ & $188(53.6 \%)$ \\
\hline \multicolumn{4}{|l|}{ Prognostic index, STarT } \\
\hline Low; n (\%) & $428(44.3 \%)$ & $89(33.8 \%)$ & $140(43.1 \%)$ \\
\hline Medium; n (\%) & $351(36.3 \%)$ & $92(35.0 \%)$ & $115(35.4 \%)$ \\
\hline High; n (\%) & $188(19.4 \%)$ & $82(31.2 \%)$ & $70(21.5 \%)$ \\
\hline Activity limitation, RMDQ (0-100); mean (SD) & $55.3(23.7)$ & $58.0(23.3)$ & $54.6(24.3)$ \\
\hline Ability to control pain, NRS $(0-10)$; mean (SD) & $5.5(2.3)$ & $5.3(2.3)$ & $5.4(2.4)$ \\
\hline
\end{tabular}

$\mathrm{NRS}=$ Numeric Rating Scale; RMDQ = Roland Morris Disability Questionnaire; STarT = The Keele STarT Back Screening Tool; SD = standard deviation

\section{Strengths and limitations}

To our knowledge, this is the first cohort of patients with musculoskeletal pain which has sufficient power and a study design making it possible to compare two otherwise similar groups with and without frequent pain monitoring. There was no difference in attrition among the two groups, but the SMS group reported slightly higher education, lower workload, and shorter baseline duration of LBP than the control group. As all three of these have been shown to predict a better prognosis [20, 21], it cannot be ruled out that group differences reflected in these characteristics could explain (part of) the better pain outcome observed in the SMS group. However, we believe the influence of these factors are limited as the outcome (pain intensity, activity limitation and ability to control the pain) were similarly distributed among the two groups at baseline, and the analyses were adjusted for relevant baseline factors. We did not find matching of cases and controls justified as the potential advantages are likely to be minimal, since group allocation occurred through a non-selective chronological mechanism, and baseline differences between the two groups were minor.

The two groups were sampled in the same manner, since they were included in the same cohort, but the control group followed the SMS group in time. This time difference could potentially lower the credibility of comparisons but there is no reason to believe that this is the case, since there was no time gap between sampling of the two groups. Sampling simply continued, just without SMS-tracking, when the required number was 
reached for the SMS-tracking. Furthermore, there were no changes in treatment recommendations, reimbursement or administrative procedures in health care during the total sampling period (2016-2018).

\section{Implications for management}

Remote patient monitoring is proposed as a tool to facilitate communication between patient and health care provider in order to improve timeliness, patient autonomy, and quality of care [22-24], and pain monitoring is common in pain management apps [8,9]. However, in the case of LBP there is still not much evidence to guide a response to changes in pain reporting, although the field of remote monitoring and telemedicine is also expanding for LBP management $[25,26]$ and intelligent decision support systems are being developed [27]. For the time being, it seems that the simple advice to systematically monitor pain could potentially prove helpful for patients with LBP. It is possible that self-monitoring leads to selfreflection, which in turn might support helpful habits and facilitate positive behavior changes.[5]. However, despite emerging evidence for effectiveness of e-health supported self-management in LBP [28], currently there is no evidence to suggest how the element of pain tracking may improve self-management and health care for LBP. Our results support continued research into this field as the intervention seems free of general negative side effects.

\section{Implications for research}

Continuous registration of signs and symptoms are important to understand disorders such as LBP and many other musculoskeletal complaints, which demonstrate intermittent and recurrent patterns. This could cause some concern as a continuous focus on pain might be feared to affect pain perceptions and pain behaviour unfavourably, and therefore some researchers might refrain from continued monitoring. However, our results suggest that concerns related to a potential risk of negative impact are unfounded. On the other hand, as the monitoring itself may have a small positive effect on pain intensity, cohorts subjected to frequent follow-up/continued monitoring should not be regarded as untreated or representing the true natural course of a disorder. In this study no feedback was provided to patients as part of pain monitoring, and it is a topic for future research to identify useful content and formats of such feedback.

\section{Conclusion}

We found no evidence that frequent self-reporting of pain has a negative effect on patient reported outcomes in patients with low back pain. On the contrary, we observed slightly better pain intensity outcomes after one year in patients with weekly monitoring, whereas no effects were observed on activity limitation and pain control. The role of self-monitoring as part of self-managing LBP should be investigated further.

\section{Abbreviations \\ ChiCo: Chiropractic Cohort; ÖMPQ: Örebro Musculoskeletal Pain Question- naire; OPEN: Open Patient data Explorative Network; LBP: Low Back Pain; REDCap: Research Electronic Data Capture; SMS: Short Text Message.}

\section{Acknowledgements}

The participating patients, chiropractors, and practice staff are acknowledged for their contribution to the study. The Odense Patient data Explorative Network (OPEN) is acknowledged for providing access to REDCap, data storage and technical support.

\section{Authors' contributions}

$A K, K D$ and TSJ took part in planning and conducting the data collection. $\mathrm{LH}, \mathrm{AK}, \mathrm{KD}$ and TSJ contributed to study design, interpretation of results, and provided feedback to the draft manuscript. AK drafted the manuscript and conducted data analyses. LH, AK, KD and TSJ all approved the final version of the manuscript.

\section{Funding}

The ChiCo cohort was financed by a grant from the Danish Foundation for Chiropractic. Research and Post Graduate Education (Document No. 21927/16). The funder was not involved in defining the research questions, designing the study, analyses of data, or interpretation of results.

\section{Availability of data and materials}

The data is available from the corresponding author on reasonable request.

\section{Declarations}

Ethics approval and consent to participate

All methods were carried out in accordance with relevant guidelines and regulations. The data collection was approved by the Danish Data Protection Agency as part of University of Southern Denmark's joint registration (Journal no. 2015-57-0008). All patients were informed about the project and their rights and provided informed consent that data would be used for research purposes. According to Danish law, the study did not require research ethics approval (Research Ethical Committee for Region of Southern Denmark journal no. S-20162000-109).

\section{Consent for publication \\ Not applicable.}

\section{Competing interests}

The authors declare that they have no competing interests. AK's and LH's positions at University of Southern Denmark are partly funded by the Danish Foundation for Chiropractic Research and Post Graduate Education.

\section{Author details}

${ }^{1}$ Chiropractic Knowledge Hub, Campusvej 55, 5230 Odense M, Denmark. ${ }^{2}$ Department of Sports Science and Clinical Biomechanics, University of Southern Denmark, Campusvej 55, 5230 Odense M, Denmark. ${ }^{3}$ Diagnostic Center, Silkeborg Regional Hospital, Falkevej 1, 8600 Silkeborg, Denmark.

Received: 28 April 2021 Accepted: 6 September 2021

Published online: 16 September 2021

\section{References}

1. Vos T, Lim SS, Abbafati C, Abbas KM, Abbasi M, Abbasifard M, Abbasi-Kangevari M, Abbastabar H, Abd-Allah F, Abdelalim A, Abdollahi M. Global burden of 369 diseases and injuries in 204 countries and territories, 
1990-2019: a systematic analysis for the Global Burden of Disease Study 2019. Lancet. 2020;396(10258):1204-22.

2. Cieza A, Causey K, Kamenov K, Hanson SW, Chatterii S, Vos T. Global estimates of the need for rehabilitation based on the Global Burden of Disease study 2019: a systematic analysis for the Global Burden of Disease Study 2019. Lancet. 2021;396(10267):2006-17.

3. Schulman-Green D, Jaser S, Martin F, Alonzo A, Grey M, McCorkle R, et al. Processes of self-management in chronic illness. J Nurs Scholarsh. 2012;44(2):136-44.

4. Du S, Hu L, Dong J, Xu G, Chen X, Jin S, et al. Self-management program for chronic low back pain: a systematic review and meta-analysis. Patient Educ Couns. 2017;100(1):37-49.

5. Slater H, Stinson JN, Jordan JE, Chua J, Low B, Lalloo C, et al. Evaluation of digital technologies tailored to support young people's self-management of musculoskeletal pain: mixed methods study. J Med Internet Res. 2020;22(6):e18315

6. Axen I, Bodin L, Bergstrom G, Halasz L, Lange F, Lovgren PW, et al. The use of weekly text messaging over 6 months was a feasible method for monitoring the clinical course of low back pain in patients seeking chiropractic care. J Clin Epidemiol. 2012;65(4):454-61.

7. Kongsted A, Kent P, Axen I, Downie AS, Dunn KM. What have we learned from ten years of trajectory research in low back pain? BMC Musculoskelet Disord. 2016;17:220.

8. Gamwell KL, Kollin SR, Gibler RC, Bedree H, Bieniak KH, Jagpal A, et al. Systematic evaluation of commercially available pain management apps examining behavior change techniques. Pain. 2021;162(3):856-65.

9. Devan H, Farmery D, Peebles L, Grainger R. Evaluation of self-management support functions in apps for people with persistent pain: systematic review. JMIR mHealth uHealth. 2019;7(2):e13080.

10. Darnall BD, Colloca L. Optimizing placebo and minimizing Nocebo to reduce pain, catastrophizing, and opioid use: a review of the science and an evidence-informed clinical toolkit. Int Rev Neurobiol. 2018;139:129-57.

11. Nicholl BI, Sandal LF, Stochkendahl MJ, McCallum M, Suresh N, Vasseljen $\mathrm{O}$, et al. Digital support interventions for the self-management of low back pain: a systematic review. J Med Internet Res. 2017:19(5):e179.

12. Hestbaek L, Saxtorph AC, Krogsgaard-Jensen CE, Kongsted A. Are frequent measurements in back pain research harmful? Two comparisons of back pain in groups with or without frequent follow-up. Chiropr Man Therap. 2018;26:51.

13. Kongsted A, Nielsen OL, Christensen HW, Hartvigsen J, Doktor K, Kent P, et al. The Danish Chiropractic Low Back Pain Cohort (ChiCo): description and summary of an available data source for research collaborations. Clin Epidemiol. 2020;12:1015-27.

14. Childs JD, Piva SR, Fritz JM. Responsiveness of the numeric pain rating scale in patients with low back pain. Spine. 2005;30(11):1331-4.

15. Lauridsen HH, Hartvigsen J, Manniche C, Korsholm L, Grunnet-Nilsson $\mathrm{N}$. Responsiveness and minimal clinically important difference for pain and disability instruments in low back pain patients. BMC Musculoskelet Disord. 2006;7:82.

16. Boonstra AM, Stewart RE, Köke AJ, Oosterwijk RF, Swaan JL, Schreurs KM, et al. Cut-off points for mild, moderate, and severe pain on the numeric rating scale for pain in patients with chronic musculoskeletal pain: variability and influence of sex and catastrophizing. Front Psychol. 2016;7:1466.

17. Albert HB, Jensen AM, Dahl D, Rasmussen MN. Criteria validation of the Roland Morris questionnaire. A Danish translation of the international scale for the assessment of functional level in patients with low back pain and sciatica. Ugeskr Laeger. 2003:165(18):1875-80.

18. Ostelo RW, Deyo RA, Stratford P, Waddell G, Croft P, Von KM, et al. Interpreting change scores for pain and functional status in low back pain: towards international consensus regarding minimal important change. Spine. 2008;33(1):90-4.

19. Linton SJ, Hallden K. Can we screen for problematic back pain? A screening questionnaire for predicting outcome in acute and subacute back pain. Clin J Pain. 1998;14(3):209-15.

20. Chen Y, Campbell P, Strauss VY, Foster NE, Jordan KP, Dunn KM. Trajectories and predictors of the long-term course of low back pain: cohort study with 5-year follow-up. Pain. 2018;159(2):252-60.

21. Artus M, Campbell P, Mallen CD, Dunn KM, van der Windt DA. Generic prognostic factors for musculoskeletal pain in primary care: a systematic review. BMJ Open. 2017:7(1):e012901.

22. Safavi KC, Driscoll W, Wiener-Kronish JP. Remote surveillance technologies: realizing the aim of right patient, right data. Right Time Anesth Analg. 2019;129(3):726-34.

23. Seppen BF, Wiegel J, L'Ami MJ, Dos Santos Rico SD, Catarinella FS, Turkstra $F$, et al. Feasibility of self-monitoring rheumatoid arthritis with a smartphone app: results of two mixed-methods pilot studies. JMIR Form Res. 2020;4(9):e20165.

24. Shah SS, Gvozdanovic A, Knight M, Gagnon J. Mobile app-based remote patient monitoring in acute medical conditions: prospective feasibility study exploring digital health solutions on clinical workload during the COVID crisis. JMIR Form Res. 2021:5(1):e23190.

25. Hermens HJ, Vollenbroek-Hutten MM. Towards remote monitoring and remotely supervised training. J Electromyogr Kinesiol. 2008;18(6):908-19.

26. Nordstoga AL, Bach K, Sani S, Wiratunga N, Mork PJ, Villumsen M, et al. Usability and acceptability of an app (SELFBACK) to support self-management of low back pain: mixed methods study. JMIR Rehabil Assist Technol. 2020;7(2):e18729.

27. Sandal LF, Øverås CK, Nordstoga AL, Wood K, Bach K, Hartvigsen J, et al. A digital decision support system (selfBACK) for improved self-management of low back pain: a pilot study with 6-week follow-up. Pilot Feasibility Stud. 2020;6:72.

28. Du S, Liu W, Cai S, Hu Y, Dong J. The efficacy of e-health in the selfmanagement of chronic low back pain: a meta analysis. Int J Nurs Stud. 2020:106:103507.

\section{Publisher's Note}

Springer Nature remains neutral with regard to jurisdictional claims in published maps and institutional affiliations.

Ready to submit your research? Choose BMC and benefit from

- fast, convenient online submission

- thorough peer review by experienced researchers in your field

- rapid publication on acceptance

- support for research data, including large and complex data types

- gold Open Access which fosters wider collaboration and increased citations

- maximum visibility for your research: over 100M website views per year

At $\mathrm{BMC}$, research is always in progress.

Learn more biomedcentral.com/submissions 